\title{
MMSE Channel Prediction Assisted Symbol-by-symbol Adaptive OFDM
}

\author{
M. Münster and L. Hanzo ${ }^{1}$ \\ Dept. of ECS, Univ. of Southampton, SO17 1BJ, UK. \\ Tel: +44-1703-593 125, Fax: +44-1703-594 508 \\ Email:lh@ecs.soton.ac.uk; http://www-mobile.ecs.soton.ac.uk
}

\begin{abstract}
Subband Adaptive Orthogonal Frequency Division Multiplexing (OFDM) constitutes an effective method of compensating for the frequency selective fading incurred by mobile receivers. The channel transfer function estimation error increases both as a function of the Doppler frequency and that of the subband modem mode signalling delay. This leads to a degradation of the system's performance. In this contribution a $2 D$-MMSE channel-predictor is investigated as a means of compensating for these effects, which incorporates the scheme proposed in [1] into an adaptive OFDM scheme.
\end{abstract}

\section{OVERVIEW}

Subband adaptive OFDM (AOFDM) has been shown to be an effective method of improving the system performance in mobile environments, where the subbands least affected by frequency-selective fading are assigned more bits per subcarrier, than the severely faded subbands [2-5]. The modulation mode assignment to be employed by the remote transmitter $\mathrm{A}$ in the next timeslot is determined by the local receiver $\mathrm{B}$ upon estimating the short-term channel quality to be experienced by the next received OFDM symbol on the basis of the most recently received OFDM symbol. Due to the channel's variation with time, there is a mismatch between the channel quality estimated by receiver B and that actually experienced by the following OFDM symbol transmitted by transmitter $\mathrm{A}$. This reduces the achievable performance gain of AOFDM compared to employing a single fixed modulation mode. Hence the application of AOFDM is confined to channel environments exhibiting relatively low Doppler frequencies, especially if no channel prediction is used. Hence, in order to support AOFDM in a broader range of mobility conditions, signal prediction techniques - which are well-known from the field of speech-coding for example - can be employed for obtaining a more accurate estimate of the channel quality in the next transmission timeslot on the basis of that in previous slots. A channel predictor assisted OFDM pre-equalization scheme was discussed in [6], while prediction assisted decision-directed channel estimation has been proposed in [7]. In this contribution we will study the performance of an AOFDM transceiver, which employs decisiondirected channel estimation and modulation mode adaptation. In the next section we will commence our discussions with an outline of the adaptive transceiver structure, while in Section III we detail the MMSE predictor's design. Finally, in Section IV the system's performance is evaluated under a variety of channel conditions.

\section{ADAPTIVE TRANSCEIVER}

The schematic of the adaptive time division duplex (TDD) transceiver employed in our simulations is shown in Figure 1. The sig-

ICC'2002, New York, USA

The financial support of the European Union under the auspices of the PanEuropean TRUST project and that of the EPSRC, Swindon UK is gratefully acknowledged

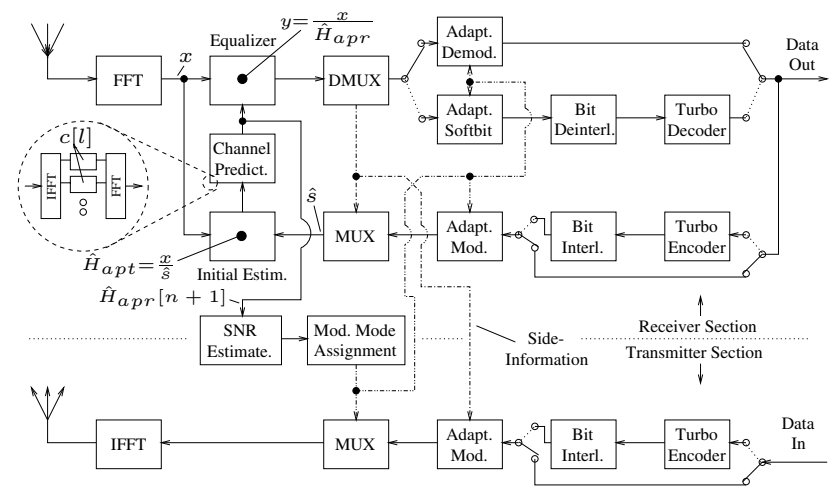

Fig. 1. Schematic of the subband adaptive transceiver employing decisiondirected MMSE channel prediction.

nal received from the remote transmitter $\mathrm{A}$ in the $n$-th downlink timeslot is forwarded to a Fast Fourier Transform (FFT) block, followed by the frequency-domain equalization of the complex symbols associated with each of the $K$ subcarriers. Equalization ensues using the á priori channel transfer factor estimates $\hat{H}_{a p r}[n, k], k=0, \ldots, K-1$ generated during the $(n-1)$-th downlink timeslot on the basis of the OFDM symbols received in the previous $P$ downlink timeslots, where each timeslot hosts one OFDM symbol. The subband modulation mode assignment to be employed by transmitter B for the OFDM symbol transmitted on the next uplink timeslot is explicitely embedded into the data stream as side-information.

As seen in Figure 1, the primary data and the modem mode signalling streams are separated from each other in the demultiplexer (DMUX) stage of Figure 1, followed by adaptive OFDM demodulation of the primary user data. Additionally, turbo coding can be employed in the system, which requires adaptive soft-bit generation at the receiver instead of direct hard-decision based adaptive demodulation, followed by channel-deinterleaving and turbo decoding. The demodulated and turbo decoded data stream is conveyed to the adaptive receiver's output. Furthermore, the sliced bits are invoked for regenerating the transmitted OFDM symbol to be used as a reference signal, which allows updating the channel transfer function estimate generated by the receiver. Hence the output bit stream has to be optionally re-encoded by the turbo encoder and re-modulated. An initial á posteriori estimate $\tilde{H}_{a p t}[n, k]$ of the actual channel transfer factor $H[n, k]$ in the $k$-th subcarrier of the $n$-th OFDM symbol is obtained upon dividing the complex received OFDM symbol $x[n, k]$ by the subcarrier's sliced symbol $\tilde{s}[n, k][1]$, yielding:

$$
\tilde{H}_{a p t}[n, k]=\frac{x[n, k]}{\tilde{s}[n, k]}, k=0, \ldots, K-1
$$




$$
=H[n, k] \cdot \frac{s[n, k]}{\tilde{s}[n, k]}+\frac{n[n, k]}{\tilde{s}[n, k]},
$$

where $H[n, k]$ denotes the Rayleigh-distributed complex channel transfer factor having a variance $\sigma_{H}^{2}$, which is unity. Furthermore, $s[n, k]$ represents the complex OFDM symbol transmitted, which exhibits zero mean and a variance of $\sigma_{s}^{2}$, and finally $n[n, k]$ is the Additive White Gaussian Noise (AWGN) contribution with a mean value of zero and variance of $\sigma_{n}^{2}$. The total noise variance $\sigma_{n}^{2}$ is constituted by the sum of the AWGN process' variance $\sigma_{A W G N}^{2}$ plus the variance of the Gaussian noise-like inter-subcarrier interference contribution $\sigma_{I C I}^{2}$. The latter component can be neglected in fading channels exhibiting a low maximum Doppler frequency $f_{D}$. However, under high-mobility channel conditions - which we envisage for our system - an estimate of $\sigma_{I C I}^{2}$ has to be provided. Upon assuming error-free symbol decisions, where $\tilde{s}[n, k]=s[n, k]$, the initial á posteriori channel transfer factor estimate of Equation 1 is simplified to:

$$
\tilde{H}_{a p t}[n, k]=H[n, k]+\frac{n[n, k]}{s[n, k]},
$$

which has a mean-square estimation error of $\beta[n, k] \sigma_{n}^{2} / \sigma_{s}^{2}$. The factor $\beta[n, k]$ depends on the modulation mode employed in the $k$-th subcarrier. For an M-PSK mode for example, $\beta[n, k]=1$, while for the 16QAM modulation mode $\beta[n, k]=17 / 9$ [8]. A 2D minimum meansquare error (MMSE) channel estimator was proposed in [1], in order to infer more accurate channel transfer factor estimates $\hat{H}_{a p t}[n, k]$ from the initial estimates $\tilde{H}_{a p t}[n, k], k=0, \ldots, K-1$. This is achieved by additionally capitalizing on the previous initial a posteriori estimates $\tilde{H}_{a p t}[n-\nu, k], \nu=1, \ldots, P-1, k=0, \ldots, K-1$ of the $(P-1)$ past OFDM symbols, where $P-1$ denotes the order of the associated estimation filter. This estimator exhibits the structure seen in Figure 1, within the circle drawn in dashed lines. Explicitely, from the frame of initial a posteriori channel transfer factor estimates $\tilde{H}_{a p t}[n, k], k=0, \ldots, K-1$ of the most recently received OFDM symbol, initially a short-term estimate of the time-variant channel impulse response (CIR) is obtained upon invoking the Inverse Fast Fourier Transform (IFFT). In the simplest case only the first $K_{0}$ CIRrelated taps' estimates are retained, while the higher-delay IFFT output samples constituted by noise - in the context of a sample-spaced CIR - are set to zero in order to reduce the noise by a factor of $K_{0} / K$. Furthermore, Wiener estimation filtering is performed [1], which will be contrasted to Wiener prediction filtering [7] in Section III. Lastly, the FFT is applied to the CIR-related tap estimates, in order to obtain the refined á posteriori estimates $\hat{H}_{a p t}[n, k]$ of the frequency domain channel transfer function of the current OFDM symbol.

In [1] these estimates were employed as á priori estimates for the frequency domain equalization of the next received OFDM symbol, neglecting the channel's Doppler-dependent transfer function variation. In channel evironments exhibiting a relatively high degree of mobility the mismatch between the channel transfer factors $\hat{H}_{a p t}[n, k], k=0, \ldots, K-1$ estimated for the current OFDM symbol, but also employed for the equalization of the next received OFDM symbol, may become excessive. Hence, in order to avoid this mismatch, the motivation of [7] was to employ a prediction filter instead of the estimation filter of [1]. This scheme was also incorporated in the adaptive transceiver structure portrayed in Figure 1.

In the proposed arrangement the advantage of employing a prediction filter is two-fold. Firstly, more accurate channel transfer factor estimates are provided for the demodulation of the next OFDM symbol received. Secondly, the channel quality expressed in terms of the signal-to-noise ratio (SNR) and potentially experienced by an OFDM symbol in the next downlink timeslot can be estimated more reliably. This is expected to enhance the performance of our AOFDM scheme. The design of the prediction filter will be further elaborated on in Section III.

The AOFDM mode adaptation performed by the modem is based on the choice between a set of four modulation modes, namely $4,2,1$ and $0 \mathrm{bit} / \mathrm{subcarrier}$, where the latter mode corresponds to 'no transmission' [2]. The modulation mode could be in theory assigned on a subcarrier-by-subcarrier basis, but the signalling overhead of such a system would be prohibitive, without significant performance advantages [2]. Hence, we have grouped adjacent subcarriers into 'subbands' and assigned the same modulation mode to all subcarriers in a subband $[2,4]$. Note that the frequency domain channel transfer function is typically not constant across the subcarriers of a subband, hence the modem mode adaptation will be sub-optimal for some of the subcarriers. The modem mode adaptation is achieved on the basis of the SNR estimated in each of the $K$ subcarriers for the OFDM symbol hosted by the $(n+1)$-th downlink timeslot, which is formulated as:

$$
\operatorname{SNR}[n+1, k]=\hat{H}_{a p r}^{2}[n+1, k] \frac{\sigma_{s}^{2}}{\sigma_{n}^{2}} .
$$

The iterative AOFDM mode assignment commences by calculating in the first step for each subband and for all four modulation modes the expected overall subband bit error ratio (BER) by means of averaging the estimated individual subcarrier BERs [2]. Throughout the second step of the algorithm - commencing with the lowest throughput but most robust modulation mode in all subbands - in each iteration the number of bits/subcarrier of that particular subband is increased, which provides the best compromise in terms of increasing the number of expected bit errors and the number of additional data bits accommodated. This process continues, until the target number of bits to be transmitted by the OFDM symbol is reached. This algorithm originates from the philosophy of the Hughes-Harthogs algorithm [9]. As a result of intensive research in the area recently several computationally efficient versions of the algorithm have emerged [2]. Again, the computed AOFDM mode assignment is explicitely signalled to the remote transmitter A on the next uplink OFDM symbol transmitted by transmitter B for employment in the forthcoming downlink timeslot. In the next section the design of the CIR-related tap predictor will be outlined.

\section{THE MMSE-PREDICTOR}

In this section we highlight the structure of the MMSE predictor $[10,11]$. Specifically, an á priori estimate $\hat{h}_{a p r}[n+1, l]$ of the $l$-th significant CIR-related tap, where $l<K_{0}$, is given by:

$$
\hat{h}_{a p r}[n+1, l]=\sum_{\nu=0}^{P-1} c[\nu, l] \cdot \tilde{h}_{a p t}[n-\nu, l],
$$

for the $(n+1)$-th timeslot. In Equation 4 the variable $c[\nu, l]$ denotes the $\nu$-th coefficient of the $P$-tap predictor and $\tilde{h}_{a p t}[n-\nu, l]$ represents the á posteriori estimate of the $l$-th CIR tap in the $(n-\nu)$-th timeslot, which is related to the a posteriori estimates $\tilde{H}_{a p t}[n-\nu, k], k=$ $0, \ldots, K-1$ of the true channel transfer factors by the Discrete Fourier Transform (DFT) matrix W. Upon invoking Equation 4, the squared error between the true CIR-related tap value $h[n+1, l]$ and the á priori estimate $\hat{h}_{a p r}[n+1, l]$ can be expressed as:

$$
|e[n, l]|^{2}=\left|h[n+1, l]-\hat{h}_{a p r}[n+1, l]\right|^{2}
$$




$$
=\left|h[n+1, l]-\sum_{\nu=0}^{P-1} c[\nu, l] \cdot \tilde{h}_{a p t}[n-\nu, l]\right|^{2} .
$$

By differentiating Equation 5 with respect to each of the CIR predictor coefficients $c[\kappa, l], \kappa \in\{0, \ldots, P-1\}$, a set of $P$ equations can be obtained, which are known in the literature as the Wiener-Hopf equations [11] given by:

$$
\begin{gathered}
\sum_{\nu=0}^{P-1} c^{*}[\nu, l] \cdot E\left\{\tilde{h}_{a p t}[n-\kappa, l] \cdot \tilde{h}_{a p t}^{*}[n-\nu, l]\right\}= \\
E\left\{\tilde{h}_{a p t}[n-\kappa, l] \cdot h^{*}[n+1, l]\right\},
\end{gathered}
$$

where again $\kappa \in\{0, \ldots, P-1\}$ and $E\{\}$ denotes the expected value. By introducing vector notation, a more convenient representation of Equation 6 can be found:

$$
\mathbf{R}_{a p t}^{[t]}[l] \cdot \mathbf{c}[l]=\mathbf{r}_{a p t}^{[t]}[l],
$$

where the $(P \times P)$-auto-correlation matrix $\mathbf{R}_{a p t}^{[t]}[l]$ of the á posteriori CIR-related tap estimates used for predicting the $l$-th CIR-related tap is given by:

$$
\mathbf{R}_{a p t}^{[t]}[l]=E\left\{\tilde{\mathbf{h}}_{a p t}[l] \cdot \tilde{\mathbf{h}}_{a p t}^{H}[l]\right\}=\mu_{l}^{[f]} \mathbf{R}^{[t]}+\frac{\sigma_{n}^{2}}{\sigma_{s}^{2}} \mathbf{I} .
$$

In Equation 8 the variable $\mu_{l}^{[f]}$ denotes the $l$-th CIR-related tap's variance, which can be expressed as the $l$-th diagonal element of the decomposition of the channel's spaced-frequency correlation ma$\operatorname{trix}^{1} \mathbf{R}^{[f]}$ with respect to the unitary DFT matrix $\mathbf{W}$, namely as $\mu_{l}^{[f]}=\left(\mathbf{W}^{H} \mathbf{R}^{[f]} \mathbf{W}\right)_{[l, l]}$. In the specific case, when the KarhunenLoeve Transform (KLT) matrix $\mathbf{U}$ - rather than the DFT matrix $\mathbf{W}$ - is employed for transforming the á posteriori channel transfer factor estimates from the frequency-domain to the CIR-related domain, the decomposition results in a diagonal shape matrix with the $l$-th element given by $\lambda_{l}^{[f]}=\left(\mathbf{U}^{H} \mathbf{R}^{[f]} \mathbf{U}\right)_{[l, l]}$, which is known as the $l$-th eigenvalue of the matrix $\mathbf{R}^{[f]}$. The eigenvectors corresponding to the different eigenvalues of $\mathbf{R}^{[f]}$ are hosted by the matrix $\mathbf{U}$. Note that in the context of the idealistic scenario of a sample-spaced CIR the DFT matrix $\mathbf{W}$ is identical to the KLT matrix $\mathbf{U}$. The application of the KLT for transforming the correlated á posteriori channel transfer factor estimates to the CIR-related domain results in a representation, where the number of significant coefficients is as low as possible. Furthermore, these coefficients are uncorrelated with each other. Despite these advantages, the application of the KLT is impractical due to the following reasons. First of all, perfect knowledge of the channel's spaced-frequency correlation matrix is required, which is normally not available. Secondly, depending on the number of significant CIR-related taps retained, namely on $K_{0}$, the associated complexity might be excessive. In Equation 8 the matrix $\mathbf{R}^{[t]}$ is the spaced-time correlation matrix, which hosts the samples of the channel's normalized spaced-time correlation function $r^{[t]}(\Delta t)$ [1]. Furthermore, note that in Equation 8 we have neglected the effects of the modulation mode-dependent SNR variations alluded to earlier in Section II. The $(P \times 1)$-cross-correlation vector $\mathbf{r}_{a p t}^{[t]}[l]$ seen in Equation 7 is defined by:

$$
\mathbf{r}_{a p t}^{[t]}[l]=E\left\{\tilde{\mathbf{h}}_{a p t}[n, l] \cdot h^{*}[n+1, l]\right\}=\mu_{l}^{[f]} \mathbf{r}^{[t]},
$$

${ }^{1}$ The channel's spaced-frequency correlation matrix is defined as $\mathbf{R}^{[f]}=$ $E\left\{\mathbf{H}[n] \mathbf{H}^{H}[n]\right\}$, where $\mathbf{H}[n]=(H[n, 0], \ldots, H[n, K-1])^{T}$. The individual matrix elements are also the samples of the channel's normalized spacedfrequency correlation function $r^{[f]}(\Delta f)[1]$. where $\mathbf{r}^{[t]}$ is the cross-correlation vector also hosting the samples of the channel's normalized spaced-time correlation function $r^{[t]}(\Delta t)$. Furthermore, $\tilde{\mathbf{h}}_{\text {apt }}[l]$ is the $(P \times 1)$-sample vector containing the $P$ ' posteriori estimates associated with the $l$-th CIR-related tap:

$$
\tilde{\mathbf{h}}_{a p t}[n, l]=\left(\tilde{h}_{a p t}[n, l], \ldots, \tilde{h}_{a p t}[n-(P-1), l]\right)^{T},
$$

and $\mathbf{c}[l]$ is the $(P \times 1)$-vector of CIR predictor coefficients:

$$
\mathbf{c}[l]=\left(c^{*}[0, l], \ldots, c^{*}[n-(P-1), l]\right)^{T} .
$$

A conceptually straightforward solution of Equation 7 for $\mathbf{c}[l]$ is given by the direct inversion of the auto-correlation matrix $\mathbf{R}_{a p t}^{[t]}[l]$, yielding:

$$
\mathbf{c}[l]=\mathbf{R}_{a p t}^{[t]-1}[l] \cdot \mathbf{r}_{a p t}^{[t]}[l] .
$$

However, computationally more efficient approaches, such as the Levinson-Durbin algorithm [11] are available, which take into account the Hermitean structure of the auto-correlation matrix that is stated as $\mathbf{R}_{a p t}^{[t] H}=\mathbf{R}_{a p t}^{[t]}$. The á priori estimation $M S E_{a p r}[l]$ of the $l$-th CIRrelated tap predictor is given by rearranging Equation 5 and calculating the expected value of $|e[n, l]|^{2}$, yielding [11]:

$$
M S E_{a p r}[l]=\mu_{l}^{[f]}-2 \mathfrak{R e}\left\{\mathbf{c}^{H}[l] \cdot \mathbf{r}_{a p t}^{[t]}[l]\right\}+\mathbf{c}^{H}[l] \cdot \mathbf{R}_{a p t}^{[t]}[l] \cdot \mathbf{c}[l],
$$

where in the context of the optimum Wiener solution of Equation 12 we obtain the following expression for the Minimum MSE (MMSE):

$$
M M S E_{a p r}[l]=\mu_{l}^{[f]}-\mathbf{c}^{H}[l] \cdot \mathbf{r}_{a p t}^{[t]}[l] .
$$

Furthermore, by capitalizing on the results of [8] the average estimation MSE observed in the frequency-domain upon retaining only the first $K_{0}$ CIR-related á priori tap estimates, while setting the remaining tap estimates equal to zero is given by:

$$
\left.\overline{M S E}_{a p r}\right|_{K_{0}}=\frac{1}{K} \sum_{l=0}^{K_{0}-1} M S E_{a p r}[l]+\frac{1}{K} \sum_{l=K_{0}}^{K-1} \mu_{l}^{[f]} .
$$

The difference between the predictor employed in our system portrayed in Figure 1 and the estimator proposed in [1] is in the definition of the cross-correlation vector of Equation 9. Specifically, the predictor employs an estimate of the cross-correlation between the $l$-th CIR tap's estimates $\tilde{h}_{\text {apt }}[n-\nu, l], \nu=0, \ldots, P-1$ of the previous $P-1$ plus the current CIRs and the actual $l$-th CIR tap $h[n+1, l]$ expected during the $(n+1)$-th OFDM symbol. By contrast, the estimator capitalizes on the cross-correlation between the $l$-th CIR tap's estimates of the previous $P-1$ plus the current CIRs and the actual $l$-th CIR tap $h[n, l]$ expected during the $n$-th OFDM symbol, which belongs to the current timeslot.

As observed in Equations 8 and 9, calculation of the auto-correlation matrix $\mathbf{R}_{a p t}^{[t]}[l]$ and the cross-correlation vector $\mathbf{r}_{a p t}^{[t]}[l]$ inherently requires knowledge of the channel's spaced-time spaced-frequency correlation function $r_{H}(\Delta t, \Delta f)$, which was shown in [1] to be separable. The separability implies that we have $r_{H}(\Delta t, \Delta f)=r^{[t]}(\Delta t)$. $r^{[f]}(\Delta f)$ under the condition that the different CIR taps' fading processes have the same correlation versus time, namely $r^{[t]}(\Delta t)$. In $[12,13]$ it was proposed by Höher et al. to employ a uniform, ideally support-limited scattering function $S_{h}\left(f_{d}, \tau\right)$ associated with 2D sinc-shaped spaced-time spaced-frequency correlation function $r_{H}(\Delta t, \Delta f)$ in the context of 2D-FIR Wiener filter-based channel estimation. The aim of this design was that of rendering the estimator insensitive against the variations of the channel's associated multipath intensity profile and Doppler power spectrum. Specifically, it 
was argued in [1] that under these conditions no further MSE performance degradation is observed for channels having a multipath spread of $T_{m}$ and Doppler spread of $B_{D}$, when the estimator is designed for $\tilde{T}_{m} \geq T_{m}$ and $\tilde{B}_{D} \geq B_{D}$. Hence this estimator was termed "robust". In this case the robust estimator would exhibit the same MSE performance, as the optimum channel estimator capitalizing on perfect knowledge of the channel's statistics in the context of a channel with a uniform, ideally support-limited scattering function. In general this is not exactly true, except for linear estimation filters of an infinite order, which hence employ an infinite number of signal samples. An exception is the idealistic scenario of encountering a sample-spaced CIR, where perfect robustness against the variations of the channel's multipath intensity profile can be achieved by capitalizing on a finite number channel transfer function samples, namely on those contained in the bandwidth of an OFDM symbol. In this case the uniform, ideally support-limited sample-spaced multipath intensity profile, which renders the estimator robust, is given by $\tilde{\mu}_{l}^{[f]}=\frac{K}{K_{0}}, l=0, \ldots, K_{0}-1^{2}$ and $\tilde{\mu}_{l}^{[f]}=0, l=K_{0}, \ldots, K-1$, where $T_{m}=K_{0} T_{s}$, which will be used as estimates of the CIR-related tap variances $\mu^{[f]}[l]$ required in Equation 8. In [1] this was also shown to be an acceptable choice in the context of non-sample-spaced CIRs. In conclusion, the uniform, ideally support-limited Doppler power spectrum and its associated spaced-frequency correlation function employed in our performance assessment in Section IV is given by:

$$
S_{H}\left(f_{d}\right)=\frac{1}{B_{D}} \operatorname{rect}\left(\frac{f_{d}}{B_{D}}\right)
$$

with:

$$
r^{[t]}(\Delta t)=\mathcal{F} \mathcal{T}^{-1}\left(S_{H}\left(f_{d}\right)\right)=\operatorname{sinc}\left(\pi B_{D} \Delta t\right) .
$$

Specifically in the context of the OFDM system considered we have $\Delta t=(\gamma-\delta) T_{t d}$, where $\gamma$ and $\delta$ are the integer indices of different OFDM symbols between which the associated channel transfer functions' time-domain correlation is to be calculated, while $T_{t d}$ denotes the difference in time between two consecutive OFDM symbols.

\section{Simulation Results}

In this section the performance of the decision-directed channel prediction assisted subband adaptive OFDM transceiver will be assessed in the context of the indoor Wireless Asynchronous Transfer Mode (WATM) channel environment characterized by the short (S)CIR of [2], which we hence refer to in the figures as the SWATM channel. In Figure 2 we have portrayed the performance of the AOFDM modem for three different normalized Doppler frequencies $F_{D}=f_{D} T_{f}$, where $T_{f}$ denotes the OFDM symbol duration. Explicitely, this implies that the Doppler frequency was normalised to the OFDM symbol duration, rather than to the time-domain sampling interval duration, typically used in single-carrier modems. Two scenarios are compared against each other, namely that of zero-delay signalling of the modulation mode assignment ('cont.') and that of a more realistic one Time Division Duplex (TDD) slot duration delay arrangement. Explicitely, the modulation mode assignment requested by receiver $\mathrm{B}$ for the next downlink timeslot was signalled by transmitter B to receiver $\mathrm{A}$ in the uplink slot. For these simulations we assumed perfect channel transfer function knowledge, zero-delay signalling of the

\footnotetext{
${ }^{2}$ This is valid when using the Hermitean transpose of the DFT matrix W for performing the transform to the CIR-related domain. However, when employing the IDFT according to standard definition, we have $\tilde{\mu}_{l}^{[f]}=\frac{1}{K_{0}}, l=$ $0, \ldots, K_{0}-1$.
}

AOFDM modulation mode assignment and 'frame-invariant' fading, where the CIR taps were kept constant for the duration of an OFDM symbol, in order to avoid inter-subcarrier interference (ICI). This allowed as to study the performance of channel prediction without the obfuscating ICI effects [2]. Upon comparing the performance curves for the idealistic scenario of zero-delay signalling with that of TDD signalling at an OFDM symbol normalized Doppler frequency of $f_{D} T_{f}=0.01$, we observe a slight BER performance degradation for the latter scenario. This is, because the time-domain separation between two consecutive OFDM symbols is effectively doubled. In other words the channel transfer function estimate employed during the current downlink OFDM symbol period for deciding upon the modulation mode assignment to be used during the following downlink OFDM symbol period is less correlated with the true channel transfer function actually experienced by this OFDM symbol. An additional BER degradation is observed upon further increasing the OFDM symbol normalized Doppler frequency. For example, at $f_{D} T_{f}=0.05$ the performance advantage in favour of the AOFDM scheme has completely diminished compared to the fixed modulation based QPSK-assisted OFDM scheme of the same throughput. This is our motivation for employing CIR-related tap prediciton filtering.

In our forthcoming simulations the idealistic assumptions of 'frame-invariant' fading and zero-delay signalling were removed, again, in favour of the 'frame-variant' fading TDD scenario. The uncoded BER performance is illustrated in Figures 3 and 4. In Figure 3 we characterised the low-mobility scenario of $f_{D} T_{f}=0.01$, while in Figure 4 the high-mobility scenario of $f_{D} T_{f}=0.05$. As a reference, we have plotted in both figures the performance exhibited by the fixed BPSK and QPSK modulation modes, employing a 4-tap predictor. Upon invoking AOFDM modulation we observed - particularly for the 4-tap CIR-related tap predictor - a tremendeous performance gain over the fixed modulation modes. The improvement was more dramatic for the high-mobility environment associated with Figure 4, where a BER floor was observed at high SNRs as a result of the fading-induced inter-subcarrier interference. In this scenario, low-BER transmission was infeasible without CIR prediction. For relatively low SNRs the BER performance was more limited as a result of erroneous symbol decisions in the initial CIR estimation stage of the decision-directed channel estimator. This effect is a consequence of transmitting training information in every 32 -th OFDM symbol only.

In conclusion, we have demonstrated that by invoking MMSE CIR prediction, efficient decision-directed channel estimation can be successfully employed in conjunction with AOFDM even under high-mobility channel conditions.

\section{REFERENCES}

[1] Y. Li, L. J. Cimini, and N. R. Sollenberger, "Robust Channel Estimation for OFDM Systems with Rapid Dispersive Fading Channels," IEEE Trans. on. Comms., vol. 46, pp. 902-915, Apr 1998.

[2] L. Hanzo, W. Webb, and T. Keller, Single- and Multi-carrier Quadrature Amplitude Modulation. IEEE Press- John Wiley, April 2000.

[3] L. Hanzo, C. Wong, and M. Yee, Adaptive Wireless Transceivers. John Wiley, IEEE Press, 2002. (For detailed contents, please refer to http://www-mobile.ecs.soton.ac.uk.).

[4] T. Keller and L. Hanzo, "Adaptive multicarrier modulation: A convenient framework for time-frequency processing in wireless communications," Proceedings of the IEEE, vol. 88, pp. 611-642, May 2000.

[5] H. Rohling and R. Gruenheid, "Adaptive Coding and Modulation in an OFDM-TDMA Communication System," in Vehicular Technology Conference, vol. 2, (Ottawa, Canada), pp. 773-776, IEEE, May 18-21 1998. 


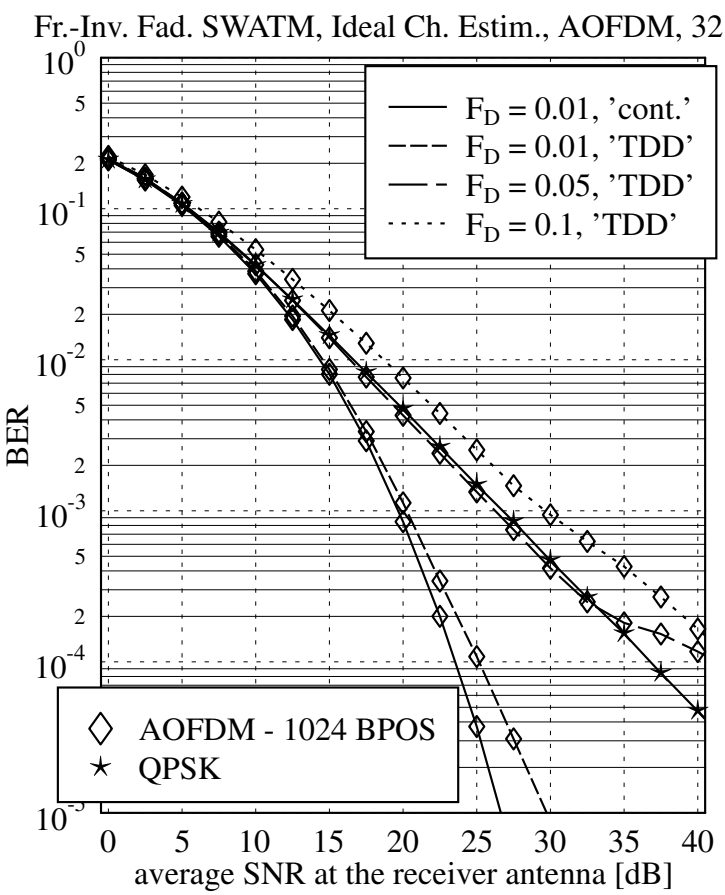

Fig. 2. Influence of the normalized Doppler frequency and the modulation mode signalling delay on the BER performance of the 32-subband 512 -subcarrier AOFDM modem in the 'frame-invariant' fading indoor SWATM environment [2] assuming perfect channel transfer function knowledge. The AOFDM mode signalling delay was 0 ('cont.') or 1 AOFDM symbol duration (TDD) and 1024 Bits Per OFDM Symbol (BPOS) were transmitted.

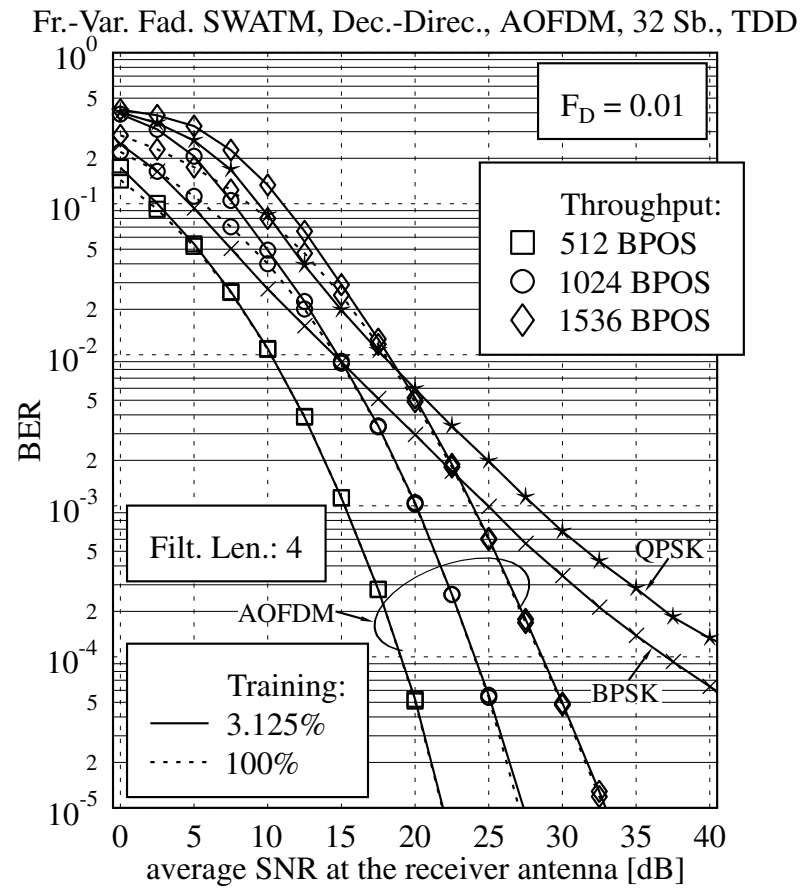

Fig. 3. Influence of the predictor length and the AOFDM throughput (Bits Per OFDM Symbol) on the BER performance of the decision-directed channel prediction assisted 32-subband 512-subcarrier AOFDM modem in the 'frame-variant' fading indoor SWATM environment [2] exhibiting a normalized Doppler frequency of $f_{D} T_{f}=0.01$. The number of significant CIR-related taps was $K_{0}=12$.
Fr.-Var. Fad. SWATM, Dec.-Direc., AOFDM, 32 Sb., TDD

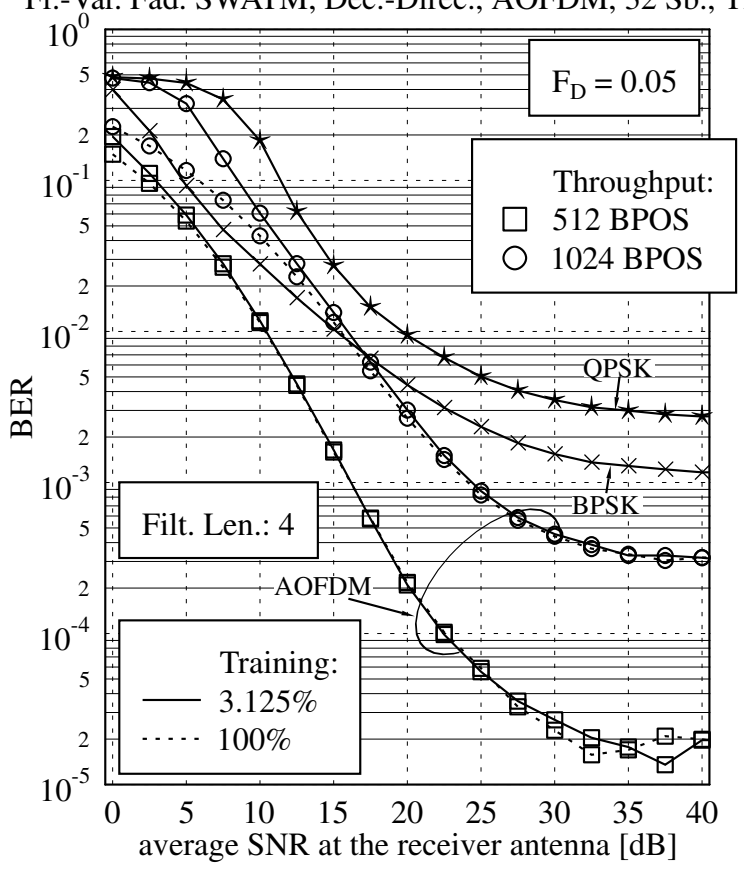

Fig. 4. Influence of the predictor length and the AOFDM throughput on the BER performance of the decision-directed channel prediction assisted 32 -subband 512-subcarrier AOFDM modem in the 'frame-variant' fading indoor SWATM environment [2] exhibiting a normalized Doppler frequency of $f_{D} T_{f}=0.05$. The number of significant CIR-related taps was $K_{0}=12$.

[6] F. Tufvesson, M. Faulkner, and T. Maseng, "Pre-Compensation for Rayleigh Fading Channels in Time Division Duplex OFDM Systems," in Proc. of 6th International Workshop on Intelligent Signal Processing and Communications Systems, (Melbourne, Australia), pp. 57-33, IEEE, November 5-6 1998.

[7] E. Al-Susa and R. F. Ormondroyd, "A Predictor-Based Decision Feedback Channel Estimation Method for COFDM with High Resilience to Rapid Time-Variations," in Proc. of Vehicular Technology Conference, vol. 1, (Amsterdam, Netherlands), pp. 273-278, IEEE, September 19-22 1999.

[8] O. Edfors, M. Sandell, J.-J. v. d. Beek, S. K. Wilson, and P. O. Börjesson, "OFDM Channel Estimation by Singular Value Decomposition," IEEE Trans. on. Comms., vol. 46, pp. 931-939, Apr 1998.

[9] D. Hughes-Hartogs, "Ensemble modem structure for imperfect transmission media," U.S. Patents Nos. 4,679,222 (July 1987), 4,731,816 (March 1988) and 4,833,796 (May 1989).

[10] A. Duel-Hallen, S. Hu, and H. Hallen, "Long Range Prediction of Fading Signals," IEEE Signal Processing Magazine, vol. 17, pp. 62-75, May 2000.

[11] S. Haykin, Adaptive Filter Theory. Prentice Hall, Inc., 1996.

[12] P. Höher, S. Kaiser, and P. Robertson, "Pilot-symbol-aided channel estimation in time and frequency," in Proceedings of Global Telecommunications Conference: The Mini-Conf., (Phoenix, AZ), pp. 90-96, IEEE, IEEE, November 1997.

[13] P. Höher, S. Kaiser, and P. Robertson, "Two-dimensional pilot-symbolaided channel estimation by Wiener filtering," in International Conference on Acoustics, Speech and Signal Processing, (Munich, Germany), pp. 1845-1848, IEEE, IEEE, April 1997. 